\title{
CUPCAKES ADICIONADOS DE FARINHA DE BOCAIÚVA: caracterização físico-química e avaliação sensorial entre crianças
}

\author{
Paloma Annes Zem VIEIRA ${ }^{1}$ \\ Roberta Franciele SCHEIDT ${ }^{1}$ \\ Mirelly Marques Romeiro SANTOS ${ }^{2}$ \\ Camila Jordão CANDIDO 3 \\ Elisvânia Freitas dos SANTOS ${ }^{4}$ \\ Daiana NOVELLO 5
}

\author{
${ }^{1}$ Graduada em Nutrição, Universidade Estadual do Centro-Oeste (UNICENTRO). \\ ${ }^{2}$ Graduada em Nutrição, Universidade Federal de Mato Grosso do Sul (UFMS). \\ ${ }^{3}$ Farmacêutica, Mestre, Técnica em Alimentos e Laticínios (UFMS). \\ ${ }^{4}$ Professora, Doutora, Departamento de Nutrição (UFMS). \\ ${ }^{5}$ Professora, Doutora, Departamento de Nutrição (UNICENTRO). *E-mail: nutridai@gmail.com.
}

Recebido em: 24/04/2016 - Aprovado em: 22/11/2017 - Disponibilizado em: 30/12/2017

\begin{abstract}
RESUMO: O presente estudo objetivou verificar a aceitabilidade sensorial de cupkakes adicionados de farinha de bocaiúva (FB), e determinar a composição físico-química da formulação padrão e daquela contendo maior teor de FB e com aceitação sensorial semelhante a padrão. Foram desenvolvidas as seguintes formulações de cupkakes: F1 (padrão $0 \%$ e as demais adicionadas de 5\% (F2), 10\% (F3), 15\% (F4) e 20\% (F5) de FB. Participaram da avaliação sensorial 43 provadores não treinados, de ambos os gêneros, com idade entre 7 e 10 anos. Nas análises físico-químicas foram determinados o teor de umidade, cinzas, proteínas, lipídios, carboidratos, fibra alimentar e valor calórico. Os resultados da análise sensorial demonstraram que não houve diferença significativa $(\mathrm{p}>0,05)$ entre as formulações para os atributos avaliados (aparência, aroma, sabor, textura, cor e aceitação global e intenção de compra). A formulação F1 apresentou maiores teores de proteínas e carboidratos quando comparada a F5, sendo que conteúdos mais elevados de umidade, cinzas, lipídios, calorias e fibras foram observados em F5. Assim, a elaboração dos produtos permitiu comprovar que um nível de adição de até $20 \%$ de FB em cupkakes foi bem aceito pelos provadores infantis, obtendo-se aceitação sensorial semelhante ao produto padrão e com boas expectativas de comercialização.
\end{abstract}

Palavras-chave: Análise sensorial. Crianças. Macaúba. Nutrição.

\section{CUPCAKES ADDED BOCAIUVA FLOUR: characterization physico-chemical and sensory evaluation among children}

\begin{abstract}
This study aimed to verify the sensory acceptability cupcakes added bocaiuva flour (BF), and determine the physico-chemical composition of standard formulation and that containing more BF content and sensory acceptance like standard. Cupkakes the following formulations were developed: F1 (standard - 0\%) and the remaining added 5\% (F2), 10\% (F3), 15\% (F4) and 20\% (F5) FB. Participated in the sensory evaluation 43 untrained, of both genders, aged between 7 and 10 years. The physico-chemical analyzes were determined the moisture, ash, protein, lipids, carbohydrates, dietary fiber and caloric value. The results of sensory analysis showed no significant difference $(\mathrm{p}>0.05)$ between formulations for the evaluated attributes (appearance, aroma, taste, texture, color and overall acceptance and purchase intent). The F1 formulation showed higher protein and carbohydrates content when compared to F5, with higher contents of moisture, ash, fat, calories and fiber were observed in F5. Thus, the development of products able to prove that a level of addition of up to $20 \%$ of BF in cupkakes was well accepted by children tasters, resulting in sensory acceptance similar to the standard product with good marketing expectations.
\end{abstract}

Keywords: Sensory analysis. Children. Macauba. Nutrition. 


\section{INTRODUÇÃO}

A bocaiúva é um fruto nativo do Estado do Mato Grosso do Sul, e é típica das regiões do cerrado brasileiro. Pertence à família Palmae, sendo mais abundante a espécie Acrocomiaaculeata (Jacq.) Lodd (AQUINO et al., 2008). Os nomes populares mais conhecidos da fruta são, também, macauva, bacauva e macaúba (REIS et al., 2012).

Os frutos do cerrado possuem características físico-químicas, nutricionais e funcionais muito relevantes (ROCHA et al., 2013). Especialmente a bocaiúva, contém em 100 g de parte comestível, 404 kcal, 2,1 g de proteínas, 40,7 g de lipídeos, 13,9 g de carboidratos, 13,4 g de fibra alimentar, $67 \mathrm{mg}$ de cálcio, $306 \mathrm{mg}$ de potássio, 13,4 mg de vitamina C (TACO, 2011) e 597,50 $\mu \mathrm{g} .100 \mathrm{~g}^{-1}$ - ERA (Equivalente de atividade retinol) de vitamina A (KOPPER et al., 2009). Destacase que a polpa apresenta alto teor de $\beta$ caroteno e $\alpha$-tocoferol, conferindo ação antioxidante, anti-inflamatória e quimiopreventiva ao organismo (RAMOS et al., 2008; COSTA et al., 2012).

A bocaiúva possui propriedades sensoriais, como cor, sabor e aroma peculiares, pouco explorados na alimentação humana

(SANJINEZ-ARGANDOÑA; CHUBA, 2011). Os frutos são de coloração que variam do amarelo ao alaranjado, esféricas ou ligeiramente achatadas, com diâmetro de 2,5 a 5,0 cm.

Geralmente, as partes comestíveis da bocaiúva são a polpa e a amêndoa. Sua ingestão pode ser na forma in natura ou como ingrediente em preparações como biscoitos, sorvetes, bolos, paçoca doce e cocada (KOPPER et al., 2009; FERREIRA et al., 2013), dentre outros.

A farinha da bocaiúva é obtida artesanalmente, por meio da secagem da polpa, que é moída e peneirada, estando, então, pronta para o consumo (KOPPER et $a l .$, 2009). O benefício da utilização da farinha comparada ao fruto é que a bocaiúva surge em períodos de safra. Deste modo, a farinha vem sendo processada pela indústria para possibilitar o seu consumo mesmo durante períodos de entre safra, aumentando a comercialização (GALVANI; SANTOS, 2010). Outra vantagem no uso da farinha se deve ao fato dos frutos in natura conterem alto teor de umidade, o que os torna muito suscetíveis à deterioração por microrganismos (COELHO; WOSIACKI, 2010).

Um produto em grande evidência nacional é o bolo. Este alimento elevou seu consumo e comercialização nos últimos anos e vem ganhando grande importância mercadológica. Apesar de não ser um alimento básico, é aceito por qualquer faixa etária (ABIMA, 2013). Destaca-se que os bolos possuem uma alta aceitabilidade entre crianças em fase escolar. Este fato é marcado 
principalmente devido às crianças demonstrarem preferência inata pelo sabor doce, bem como aos alimentos de maior densidade calórica, os quais possuem boa palatabilidade e maior sensação de saciedade (MATUK et al., 2011).

O bolo é um alimento de fácil preparo e apresenta elevado potencial para adição de farinhas mistas e novos ingredientes. Assim, estes produtos tornam-se veículos adequados para sua inclusão na merenda escolar, mediante adição de ingredientes que melhorem seu perfil nutricional (FRANZEN et al., 2014).

A infância, mais especificamente o período escolar, que compreende dos 7 a 10 anos de idade, classifica-se como uma fase que requer maiores necessidades nutricionais, precedendo o estirão pubertário. Neste aspecto, a introdução de alimentos com maior aporte de nutrientes, principalmente as vitaminas e minerais, pode colaborar para reduzir o risco de futuras doenças crônicas não transmissíveis, possibilitando uma melhor qualidade de vida (FRANZEN et al., 2014).

$A$ adição de novos ingredientes em produtos alimentícios requer testes sensoriais que avaliem a aceitabilidade pelos consumidores. Estes instrumentos são fundamentais para verificar a preferência dos alimentos (CECANE, 2010). As características sensoriais dos alimentos são verificadas através de respostas, que ocorrem por meio de reações fisiológicas resultantes de estímulos. Essas sensações podem mensurar intensidade, extensão, duração, qualidade, gosto ou desgosto em relação ao produto (OLIVEIRA; RODRIGUES, 2011). Para o público infantil, estes testes são imprescindíveis, pois as crianças fazem parte do mercado consumidor, realizando compras, especialmente na escola, apresentando grande capacidade de compra e escolha (VEIGA NETO; MELO, 2013).

O objetivo deste trabalho foi avaliar a aceitabilidade sensorial de formulações de cupcakes adicionados de farinha de bocaiúva (FB) entre crianças de idade escolar, e determinar a composição físico-química do produto com maior teor de FB e aceitação semelhante ao padrão.

\section{MATERIAL E MÉTODOS}

\section{Aquisição da matéria-prima}

A maioria ingredientes foram adquiridos em supermercados localizados no município de Guarapuava, PR. Já, a FB foi adquirida na Central de Comercialização de Economia Solidária na cidade de Campo Grande, Mato Grosso do Sul.

\section{Formulações}

Foram elaboradas cinco formulações de cupcakes, sendo: F1 padrão $(0 \%)$ e as demais adicionadas de 5\% (F2), 10\% (F3), $15 \%$ (F4) e $20 \%$ (F5) de FB. Estes níveis de adição foram definidos através de testes 
sensoriais preliminares realizados com 0 produto. Além das porcentagens de farinha de bocaiúva, os ingredientes utilizados nas formulações foram: ovos $(16,28 \%)$, leite $(2,43 \%)$, óleo de soja $(13,19 \%)$, chocolate em pó $(8,42 \%)$, açúcar refinado $(2,62 \%)$, fermento químico $(2,06 \%)$ e farinha de trigo (F1: 55\%, F2: 50\%, F3: 45\%, F4: 40\% e F5: $35 \%)$.

Para a elaboração dos cupcakes, primeiramente foram batidos o óleo, os ovos e o leite em um liquidificador doméstico (Britânia $^{\circledR}$, Brasil). Em seguida, foram adicionados os demais ingredientes, até se obter uma massa homogênea (aprox. 10 minutos). A massa foi disposta em forminhas de papel (5 $\mathrm{cm}$ de diâmetro), próprias para cupcake.

As formas de papel foram dispostas em formas de silicone individuais e, logo após, organizadas em assadeiras de alumínio $(40 \times 30 \mathrm{~cm})$. Os produtos foram assados em forno convencional $\left(\right.$ Consul $^{\circledR}$, Brasil), préaquecido em temperatura média $\left(180^{\circ} \mathrm{C}\right)$, por aproximadamente 20 minutos. Após, permaneceram em temperatura ambiente (25 ${ }^{\circ} \mathrm{C}$ ) até o momento da análise sensorial.

\section{Análise sensorial}

Participaram da pesquisa 43 provadores não treinados, sendo crianças devidamente matriculadas em uma Escola Municipal de Guarapuava, PR, de ambos os gêneros, com idade entre 7 e 10 anos.
A análise sensorial foi realizada em uma sala da escola, sendo que o provador foi orientado pelas pesquisadoras para o preenchimento do questionário sem interferência nas respostas.

Foram avaliados os atributos de aparência, aroma, sabor, textura e cor. Os provadores avaliaram a aceitação das amostras através de uma escala hedônica facial estruturada mista de 7 pontos variando de 1 ("super ruim") a 7 ("super bom"), adaptada de Resurreccion (1998). Foram aplicadas também, questões de aceitação global e intenção de compra analisados através de escala estruturada de 5 pontos (1 "desgostei muito"/ "não compraria" a 5 "gostei muito"/ "compraria com certeza"), como sugerido por Minim (2010).

Os julgadores receberam uma porção de cada amostra (aproximadamente $10 \mathrm{~g}$ ), em pratos plásticos descartáveis brancos, codificados com números de três dígitos, de forma casualizada e balanceada, acompanhados de um copo de água para realização do branco. As formulações foram oferecidas aos julgadores de forma monádica sequencial.

\section{Índice de aceitabilidade (IA)}

O cálculo do IA das cinco formulações foi realizado conforme Monteiro (1984), segundo a fórmula: $I A(\%)=A \times 100 / B(A=$ nota média obtida para o produto; $B=$ nota máxima dada ao produto). 


\section{Composição físico-química}

As seguintes determinações físicoquímicas foram realizadas em triplicata na FB, na formulação padrão e naquela com maior teor de FB e com aceitação sensorial semelhante a padrão:

Umidade: foi realizada em estufa a $105^{\circ} \mathrm{C}$ até peso constante e a determinação de cinzas foi realizada em mufla $\left(550{ }^{\circ} \mathrm{C}\right)$ (AOAC, 2011); Lipídios: utilizou-se o método de extração a frio (BLIGH; DYER, 1959); Proteínas: as amostras foram avaliadas através do teor de nitrogênio total, pelo método Kjeldahl, determinado ao nível semimicro (AOAC, 2011). Utilizou-se o fator de conversão de nitrogênio para proteína de 6,25; Fibra alimentar: foi avaliada pelo cálculo teórico das formulações utilizando-se a Tabela Brasileira de Composição de Alimentos (TACO, 2011); Carboidratos: foi realizado cálculo teórico (por diferença) nos resultados das triplicatas, conforme a fórmula: $\%$ Carboidratos $=100-(\%$ umidade $+\%$ proteína $+\%$ lipídios $+\%$ cinzas $) ;$ Valor calórico total ( $\mathrm{kcal}$ ): foi utilizado cálculo teórico, de acordo com os seguintes valores: lipídios $(8,37 \mathrm{kcal} / \mathrm{g})$, proteína $(3,87 \mathrm{kcal} / \mathrm{g})$ e carboidratos $(4,11 \mathrm{kcal} / \mathrm{g})$ (MERRILL; WATT, 1973).

\section{Determinação do valor diário de referência} (VD)

O VD foi calculado em relação a $40 \mathrm{~g}$ da amostra, com base nos valores médios preconizados para crianças de 7 a 10 anos (DRI, 2005), resultando em: $1.477,00 \mathrm{kcal} /$ dia, 212,4 g/ dia de carboidratos, 52,03 g/ dia de proteínas, 60,71 g/dia de lipídios e 9,53 g/ dia de fibra alimentar.

\section{Análise estatística}

Os dados foram analisados com auxílio do software Statgraphics Plus ${ }^{\circledR}$, versão 5.1, através da análise de variância (ANOVA), sendo que a comparação de médias foi realizada pelo teste de médias de Tukey e t de student, avaliados com nível de $5 \%$ de significância.

\section{Questões éticas}

Este trabalho foi aprovado pelo Comitê de Ética em Pesquisa da UNICENTRO (nº 608.950/2014). Entretanto, como critérios de exclusão foram considerados os seguintes fatores: possuir alergia a algum ingrediente utilizado na elaboração dos cupcakes, não ser aluno da escola em questão ou não entregar o Termo de Consentimento Livre e Esclarecido (TCLE) assinado pelo responsável legal.

\section{RESULTADOS E DISCUSSÃO}

\section{Análise sensorial}

Por meio da Tabela 1 pode-se verificar o resultado da avaliação sensorial dos cupcakes padrão e acrescidos de FB. 
Tabela 1 - Médias dos testes sensoriais afetivos de aceitação e intenção de compra, realizados para as formulações de cupcakes padrão e adicionados de diferentes níveis de farinha de bocaiúva (FB)

\begin{tabular}{lccccc}
\hline $\begin{array}{l}\text { Formulações/ } \\
\text { Atributos }\end{array}$ & F1 & F2 & F3 & F4 & F5 \\
& Média $\pm E P M$ & Média $\pm E P M$ & Média $\pm E P M$ & Média $\pm E P M$ & Média $\pm E P M$ \\
\hline Aparência & $5,81 \pm 0,23^{\mathrm{a}}$ & $5,65 \pm 0,26^{\mathrm{a}}$ & $5,48 \pm 0,27^{\mathrm{a}}$ & $5,67 \pm 0,25^{\mathrm{a}}$ & $5,69 \pm 0,22^{\mathrm{a}}$ \\
Aroma & $6,09 \pm 0,17^{\mathrm{a}}$ & $5,69 \pm 0,24^{\mathrm{a}}$ & $5,46 \pm 0,26^{\mathrm{a}}$ & $5,35 \pm 0,24^{\mathrm{a}}$ & $5,40 \pm 0,26^{\mathrm{a}}$ \\
Sabor & $6,12 \pm 0,18^{\mathrm{a}}$ & $6,14 \pm 0,18^{\mathrm{a}}$ & $5,69 \pm 0,29^{\mathrm{a}}$ & $5,67 \pm 0,28^{\mathrm{a}}$ & $5,76 \pm 0,23^{\mathrm{a}}$ \\
Textura & $5,88 \pm 0,16^{\mathrm{a}}$ & $5,12 \pm 0,29^{\mathrm{a}}$ & $5,05 \pm 0,31^{\mathrm{a}}$ & $5,39 \pm 0,27^{\mathrm{a}}$ & $5,18 \pm 0,27^{\mathrm{a}}$ \\
Cor & $5,83 \pm 0,20^{\mathrm{a}}$ & $6,04 \pm 0,19^{\mathrm{a}}$ & $5,41 \pm 0,28^{\mathrm{a}}$ & $5,55 \pm 0,25^{\mathrm{a}}$ & $6,06 \pm 0,19^{\mathrm{a}}$ \\
Aceitação global & $4,60 \pm 0,11^{\mathrm{a}}$ & $4,16 \pm 0,20^{\mathrm{a}}$ & $4,09 \pm 0,18^{\mathrm{a}}$ & $4,12 \pm 0,17^{\mathrm{a}}$ & $4,25 \pm 0,15^{\mathrm{a}}$ \\
Intenção de compra & $4,49 \pm 0,12^{\mathrm{a}}$ & $4,16 \pm 0,18^{\mathrm{a}}$ & $4,32 \pm 0,16^{\mathrm{a}}$ & $4,06 \pm 0,20^{\mathrm{a}}$ & $4,13 \pm 0,18^{\mathrm{a}}$ \\
\hline
\end{tabular}

Letras diferentes na linha indicam diferença significativa pelo teste de Tukey ( $\mathrm{p}<0,05)$; EPM: erro padrão da média; $\mathrm{F} 1$ : padrão (0\%); F2: 5\% de FB; F3: 10\% de FB; F4: 15\% de FB; F5: 20\% de FB; Fonte: os autores.

Não houve diferença significativa entre as formulações em nenhum dos atributos avaliados, bem como para a aceitação global e intenção de compra. Resultados que corroboram com Kooper et al. (2009), que avaliaram biscoitos acrescidos de FB (10 e 15\%), entre adultos. Este fato pode ser explicado porque a bocaiúva possui baixa acidez, o que favorece suas características sensoriais, tornando-a mais agradável ao paladar (MOOZ et al., 2012).

Apesar de não se verificar diferença estatística entre as formulações, foi possível verificar que os produtos contendo maior teor de FB, principalmente F5, apresentaram mudanças na aparência, textura e cor. Assim, os cupcakes se mostraram visualmente mais úmidos, com menor volume e com uma coloração mais clara. Este fato se deve, principalmente, as menores quantidades de farinha de trigo adicionadas a essas amostras.

Segundo Preichardt et al. (2013), o glúten presente na farinha de trigo, caracterizado como um conjunto de proteínas (glutenina e gliadina), promove a extensão e elasticidade das massas, retendo $\mathrm{o}$ ar $\mathrm{e}$ incorporando volume aos produtos, efeito que foi reduzido nos produtos contendo menores teores de farinha de trigo.

A Figura 1 apresenta a distribuição dos provadores pelos valores hedônicos para cada atributo sensorial. 

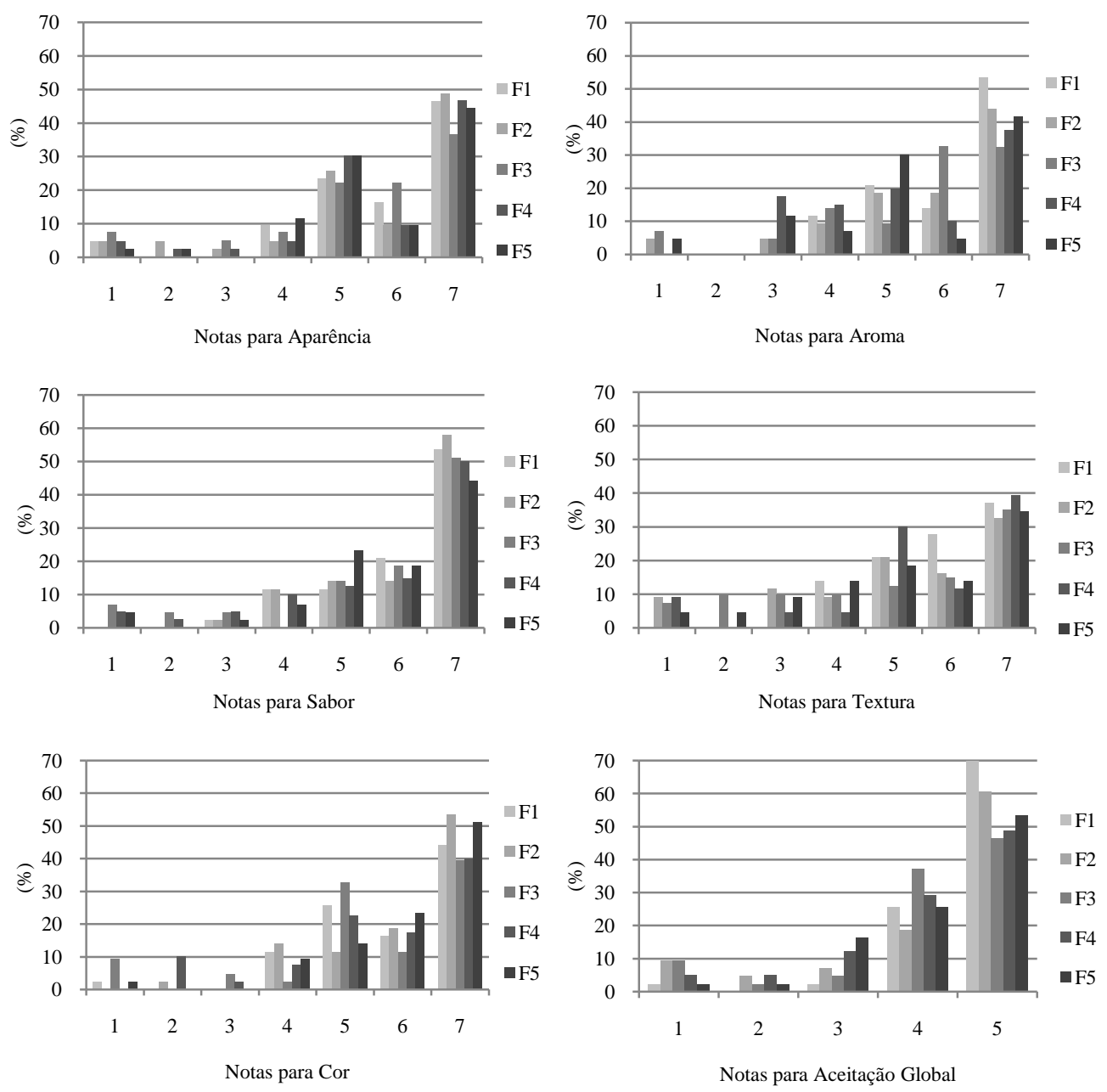

Fonte: os autores.

Figura 1 - Distribuição dos provadores pelos valores hedônicos obtidos na avaliação dos atributos aparência, aroma, sabor, textura, cor e aceitação global dos cupcakes padrão (F1) e adicionados de $5 \%(\mathrm{~F} 2), 10 \%(\mathrm{~F} 3), 15 \%$ (F4) e $20 \%$ (F5) de farinha de bocaiúva.

Verifica-se que as notas, para todos os atributos e formulações, se concentraram em 7 ("super bom"), sendo que na aceitação global a nota mais citada foi 5 ("gostei muito"). Resultados similares foram observados por Kooper et al. (2009), obtendo uma frequência de $67 \%$ para a nota 6,0 ("gostei levemente").

Em geral, constatou-se menores notas para os produtos contendo maiores teores de
FB. Alguns fatores podem explicar este efeito, dentre eles a alteração da cor causada pela adição de $\mathrm{FB}$, pois é um atributo fundamental para o julgamento da qualidade de um alimento (SANJUNEZ-ARGADONÃ; CHUBA, 2011). Além disso, a FB apresenta elevado teor de fibras, resultando em maior retenção de umidade (KOOPER et al., 2009), o que pode ter alterado a aparência geral dos produtos. 
Por meio da Figura 2 verifica-se o IA

das formulações dos cupcakes padrão e daqueles adicionados de FB em relação aos atributos avaliados.

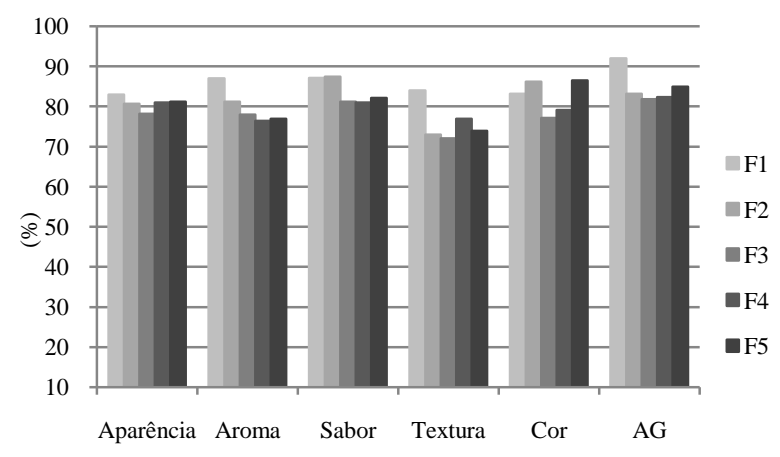

Fonte: os autores.

Figura 2 - Índice de aceitabilidade das formulações de cupcakes padrão (F1) e adicionadas de 5\% (F2), 10\% (F3), 15\% (F4) e 20\% (F5) farinha de bocaiúva, em relação aos atributos avaliados.

Segundo Teixeira et al. (1987), índices de aceitabilidade acima de $70 \%$ classificam o produto com boa aceitação sensorial, assim as 5 formulações de cupcakes adicionadas de FB podem ser classificadas como bem aceitas pelos provadores. Ressalta-se que os altos índices de aceitação das formulações adicionadas de FB são favoráveis nutricionalmente.

A ingestão de produtos contendo altos teores em fibras na infância pode colaborar para a prevenção da obesidade, além da redução do risco de outras doenças crônicas não transmissíveis como a diabetes mellitus tipo 2 (MELLO; LAAKSONEN, 2009). Além disso, as fibras também promovem benefícios ao organismo humano, aumentando o bolo fecal e atenuando o colesterol sanguíneo (MACHADO; CAPELARI, 2010; BERNAUD; RODRIGUES, 2013).

Em razão da aceitabilidade similar dos produtos, em todos os atributos e formulações, a amostra F5 (20\% de FB) foi selecionada para fins de comparação, juntamente com a padrão (F1), por ser aquela com o maior teor de FB.

\section{Composição físico-química}

Por meio da Tabela 2 pode-se observar os valores da composição físico-química dos cupcakes padrão e acrescido de $20 \%$ de farinha de bocaiúva, comparados a um produto de referência. 
Tabela 2 - Composição físico-química e valores diários recomendados - VD* (porção média de 40 gramas - 1 unidade) da farinha de bocaiúva, do cupcake padrão (F1) e daquele adicionado de $20 \%$ de farinha de bocaiúva (F5), comparadas com um produto de referência

\begin{tabular}{lcccccc}
\hline Avaliação & $\begin{array}{c}\text { Farinha de bocaiúva } \\
\text { Média } \pm \mathrm{DP}\end{array}$ & $\begin{array}{c}\text { F1 } \\
\text { Média } \pm \mathrm{DP}\end{array}$ & $\begin{array}{c}\text { VD } \\
(\%)^{*}\end{array}$ & $\begin{array}{c}\text { F5 } \\
\text { Média } \pm \mathrm{DP}\end{array}$ & \multicolumn{2}{c}{ VD } \\
& $(\%)^{*}$ & \\
\hline Umidade $(\%)$ & $8,67 \pm 0,06$ & $16,51 \pm 0,08^{\mathrm{b}}$ & $\mathrm{ND}$ & $18,35 \pm 0,01^{\mathrm{a}}$ & $\mathrm{ND}$ & $\mathrm{ND}$ \\
Cinzas $\left(\mathrm{g} \cdot 100 \mathrm{~g}^{-1}\right)^{* * *}$ & $3,31 \pm 0,03$ & $1,04 \pm 0,02^{\mathrm{b}}$ & $\mathrm{ND}$ & $1,56 \pm 0,03^{\mathrm{a}}$ & $\mathrm{ND}$ & $\mathrm{ND}$ \\
Proteínas $\left(\mathrm{g} .100 \mathrm{~g}^{-1}\right)^{* * *}$ & $3,29 \pm 0,07$ & $5,26 \pm 0,03^{\mathrm{a}}$ & 4,04 & $3,63 \pm 0,02^{\mathrm{b}}$ & 2,79 & 6,86 \\
Lipídios $\left(\mathrm{g} \cdot 100 \mathrm{~g}^{-1}\right)^{* * *}$ & $13,15 \pm 0,04$ & $7,57 \pm 0,05^{\mathrm{b}}$ & 4,99 & $11,93 \pm 0,08^{\mathrm{a}}$ & 7,86 & 6,86 \\
Carboidratos $\left(\mathrm{g} \cdot 100 \mathrm{~g}^{-1}\right) * * *$ & $71,58 \pm 0,15$ & $69,62 \pm 0,10^{\mathrm{a}}$ & 13,11 & $64,53 \pm 0,12^{\mathrm{b}}$ & 12,15 & 77,14 \\
Calorias $\left(\mathrm{kcal} .100 \mathrm{~g}^{-1}\right)^{* * *}$ & $417,01 \pm 0,45$ & $369,89 \pm 0,07^{\mathrm{b}}$ & 10,02 & $379,12 \pm 0,46^{\mathrm{a}}$ & 10,27 & 397,00 \\
Fibra alimentar $\left(\mathrm{g} \cdot 100 \mathrm{~g}^{1}\right)^{* * * *}$ & 13,40 & 1,69 & 7,14 & 3,91 & 16,53 & 3,00
\end{tabular}

Letras diferentes na linha indicam diferença significativa pelo teste de t de student (p<0,05); *VD: nutrientes avaliados pela média da DRI (2005), com base numa dieta de $1.477,00 \mathrm{kcal} / \mathrm{dia}$; **Valores comparados com um produto comercial "bolo de chocolate", marca líder de mercado; ***Valores calculados em base úmida; ****Cálculo teórico (TACO, 2011); DP: desvio padrão da média; ND: não disponível; Fonte: os autores.

Resultados similares ao presente estudo, para avaliação de FB, foram observados por Kooper et al. (2009) quanto aos teores de umidade $(9,85 \%)$, cinzas $(3,47 \%)$ e proteínas $(3,18 \%)$. Entretanto, maiores conteúdos de lipídios $(25,04 \%)$ e fibras $(22,71 \%)$ e menores de calorias $(381 \%)$ e carboidratos $(35 \%)$ foram constatados pelos autores. Destaca-se, porém, que conforme a Portaria $\mathrm{n}^{\circ} 27$ de 13 de janeiro de 1998 (BRASIL, 1998), a farinha não pode ser considerada uma fonte proteica.

$\mathrm{O}$ teor de umidade verificado na $\mathrm{FB}$ pode ser considerado seguro, pois farinhas com umidade acima de $15 \%$ podem apresentar maior probabilidade para formação de grumos, prejudicando a produção de massas, apresentando também pouca aeração, difícil conservação e mastigação (BRASIL, 2005; ESTELLER; LANNES, 2005).
Maiores teores de umidade foram encontrados na formulação F5. Este resultado pode ser explicado pela maior quantidade de fibras presentes na $\mathrm{FB}$, as quais possuem elevada capacidade de retenção de água (KOOPER et al., 2009). Estes dados corroboram com Kaefer et al. (2013), que analisaram bolos com adição de farinha de pupunha (15 e 25\%). De forma similar, na avaliação de cinzas e lipídeos foram verificados maiores conteúdos para F5. Isso se deve ao fruto apresentar altos teores de cinzas e lipídios em sua composição (Tabela 2), comparados à farinha de trigo $(1,4$ e $0,8 /$ $100 \mathrm{~g}$, respectivamente) (TACO, 2011).

A formulação padrão apresentou maiores teores de proteínas e carboidratos que F5. A explicação está na maior quantidade desses nutrientes presentes na farinha de trigo $(9,8 \quad \mathrm{~g} \quad / 100 \quad \mathrm{~g} \quad \mathrm{e} \quad 75,1 \quad \mathrm{~g} \quad / 100 \quad \mathrm{~g}$, respectivamente) (TACO, 2011) em relação à 
FB (Tabela 2). Destaca-se que o cupcake adicionado de FB é composto por um maior teor de carboidratos complexos, os quais apresentam maiores benefícios à saúde infantil. É possível constatar, também, que o conteúdo calórico foi maior em F5, o que é explicado pela menor quantidade de calorias presentes na farinha de trigo $(360 \mathrm{kcal} / 100 \mathrm{~g})$ (TACO, 2011).

De um modo geral, as formulações elaboradas apresentaram valores inferiores ao produto referência para proteínas, carboidratos e calorias. Essa diferença é caracterizada pelos diversos ingredientes utilizados nas formulações.

Destaca-se como principal resultado desse trabalho o teor de fibras verificado na formulação de cupcake adicionada de FB (F5), expressando um aumento significativo de $131,36 \%$ em relação a F1. Isso se deve, principalmente, ao alto teor de fibras presente na FB $\left(13,40\right.$ g. $\left.100 g^{1}\right)$, teor superior ao encontrado na farinha de trigo comum $(2,3$ g. $100 \mathrm{~g}^{1}$ ) (TACO, 2011). Estes resultados tornam os cupcakes elaborados com FB uma boa fonte de fibras para as crianças, visto que nesta fase há um baixo consumo deste nutriente (MELLO et al., 2010).

De acordo com a Legislação Brasileira (2012), um produto é considerado como fonte de fibra alimentar quando apresentar no mínimo $3 \%$ e com alto teor no mínimo $6 \%$ em fibras. Assim, pode-se considerar F2 como um produto fonte de fibra alimentar.

\section{CONCLUSÃO}

A elaboração dos produtos permitiu comprovar que um nível de adição de até $20 \%$ de farinha de bocaiúva em cupcakes (redução de $36,4 \%$ de farinha de trigo refinada), foi bem aceito pelos provadores, obtendo-se aceitação sensorial semelhante ao produto padrão.

A adição de $20 \%$ de farinha de bocaiúva em cupcakes elevou os teores de nutrientes, com exceção das proteínas e carboidratos. Foi possível também, elevar o aporte de fibras, melhorando o perfil nutricional do produto. Assim sendo, a bocaiúva pode ser considerada um potencial ingrediente para adição em bolos e similares, podendo ser oferecidos aos consumidores infantis com altas expectativas de aceitação no mercado.

\section{REFERÊNCIAS}

AOAC International. Official Methods of Analysis of AOAC International. 18 ed. 4 rev. Gaithersburg: AOAC, 2011. 1505p.

AQUINO, F.G.; RIBEIRO, J.F.; GULIAS, A.P.S.M.; OLIVEIRA, M.C.; BARROS, C. J. S.; HAYES, K.M.; SILVA, M.R. Uso sustentável das plantas nativas do Cerrado: oportunidades e desafios. In: PARRON, L.M. et al. Cerrado: desafios e oportunidades para o desenvolvimento sustentável. Planaltina: Embrapa Cerrados, 2008. p. 95123.

ASSOCIAÇÃO BRASILEIRA DAS INDÚSTRIAS DE MASSAS ALIMENTÍCIAS (ABIMA). Vendas de bolos no Brasil. 2013. Disponível em: 
http://www.abima.com.br/. Acesso em:

26/03/2016.

BERNAUD, F.S.R.; RODRIGUES, T.C. Fibra alimentar - Ingestão adequada e efeitos sobre a saúde do metabolismo. Arquivos Brasileiros de Endocrinologia \& Metabologia, v.57, n.6, p.397-405, 2013.

BLIGH, E.G.; DYER, W.J. A rapid method of total lipid extraction and purification.

Canadian Journal Biochemistry and

Physiology, v.37, n.8, p.911-917, 1959.

BRASIL. Agência Nacional de Vigilância Sanitária. RDC n ${ }^{\circ} \mathbf{2 6 3}$ de 22 de setembro de 2005. "Regulamento técnico para produtos de cereais, amidos, farinhas e farelos". Diário Oficial [da] República Federativa do Brasil, Brasília, DF, 2005.

BRASIL. Ministério da Saúde. Agência Nacional de Vigilância Sanitária. ANVISA. Resolução da Diretoria Colegiada - RDC no 54, de 12 de novembro de 2012. Aprova: "Regulamento Técnico sobre Informação Nutricional". Disponível em:

http://portal.anvisa.gov.br/wps/wcm/connect/ 630a98804d7065b981f1e1c116238c3b/Resol ucao+RDC+n.+54_2012.pdf?MOD=AJPERE S. Acesso em: 05/04/2016.

BRASIL. Ministério da Saúde. Portaria $\mathbf{n}^{\mathbf{0}}$. 27, de 13 de janeiro de 1998. Regulamento técnico referente à informação nutricional complementar (declarações relacionadas ao conteúdo de nutrientes) Diário Oficial [da] República Federativa do Brasil. Brasília, DF, 1998 jan. 13; Seção 1.

CENTRO COLABORADOR EM ALIMENTAÇÃO E NUTRIÇÃO ESCOLAR (CECANE). Manual para aplicação dos testes de aceitabilidade no Programa Nacional de Alimentação Escolar. São Paulo: Unifesp, 2010. 56p.

COELHO, L.; WOSIACKI, G. Avaliação sensorial de produtos panificados com adição de farinha de bagaço de maçã. Ciência e Tecnologia de Alimentos, v.30, n.3, p.582588, 2010.
COSTA, G.L.A.; MORENO, S.E.M.; FAVARO, S.P. Avaliação do potencial mutagênico, antimutagênico e antioxidante do óleo da polpa de Acrocomiaaculeata (Arecaceae). Dissertação (Mestrado em Biotecnologia aplicada à saúde) Universidade Católica Dom Bosco, Campo Grande-MS, 2012.

DIETARY REFERENCE INTAKES (DRI). Dietary Reference Intakes for energy, carbohydrate, fiber, fat, fatty acids, cholesterol, protein and amino acids. Washington: The National Academies Press, 2005. 1331p.

ESTELLER, M.S.; LANNES, S.C.S. Parâmetros Complementares para Fixação de Identidade e Qualidade de Produtos Panificados. Sociedade Brasileira de Ciência e Tecnologia de Alimentos, v.25, n.4, p.802-806, 2005.

FERREIRA, A.N.; SANTOS, C.P.A.; COSTA, G.L.A.; GEBARA, K.S. Utilização do extrato de bocaiúva (acrocomia aculeata) como um alimento funcional do tipo "Shake". Interbio, v.7, n.1, p.61-71, 2013.

FRANZEN, J.M.; NUNES, T.R.G.; FOPPA, T.; ZANCANARO, V. Elaboração e análise sensorial de cupcakes preparados a partir da farinha de semente de abóbora (curcubitamaxima) em crianças de 7 anos de idade. Revista Interdisciplinar de Estudos em Saúde, v.3, n.1, p.7-12, 2014.

GALVANI, F.; SANTOS, J.F. Extração mecânica da polpa da bocaiúva voltada para a fabricação de alimentos em comunidades extrativistas de Miranda, MS. Resumos do III Seminário de Agroecologia de MS. Cadernos de Agroecologia, v.5, n.1, p.1-4, 2010.

KAEFER, S.; FOGAÇA, A.; STORCK, C.R.; KIRSTEN, V.R. Bolo com farinha de pupunha (Bactris gasipaes): Análise da composição centesimal e sensorial.

Alimentos e Nutrição, v.24, n.3, p.347-352, 2013. 
KOPPER, A.C.; SARAVIA, A.P.K.; RIBANI, R.H.; LORENZI, G.M.A.C. Utilização tecnológica da farinha de bocaiuva na elaboração de biscoitos tipo cookie. Alimentos e Nutrição, v.20, n.3, p.463-470, 2009.

MACHADO, W.M.; CAPELARI, S.M. Avaliação da eficácia e do grau de adesão ao uso prolongado de fibra dietética no tratamento da constipação intestinal funcional. Revista de Nutrição, v.23, n.2, p.231-238, 2010.

MATUK, T.T.; STANCARI, P.C.S.; BUENO, M.B.; ZACCARELLI, E.M. Composição de lancheiras de alunos de escolas particulares de São Paulo. Revista Paulista de Pediatria, v.29, n.2, p.157-163, 2011.

MELLO, C.S.; FREITAS, K.D.C.; TAHAN, S.; MORAIS, M.D.B. Consumo de fibra alimentar por crianças e adolescentes com constipação crônica: influência da mãe ou cuidadora e relação com excesso de peso. Revista Paulista Pediatria, v.28, n.2, p.188193, 2010.

MELLO, V.D.; LAAKSONEN, D. Fibras na dieta: tendências atuais e benefícios à saúde na síndrome metabólica e no diabetes melito tipo 2. Arquivos Brasileiros de

Endocrinologia \& Metabologia, v.53, n.5, p.509-518, 2009.

MERRIL, A.L.; WATT, B.K. Energy value of foods: basis and derivation. Washington: United States Department of Agriculture, 1973. 105p.

MINIM, V.P.R. Análise Sensorial: estudo com consumidores. 2 ed. Viçosa: UFV, 2010. 308p.

MONTEIRO, C.L.B. Técnicas de avaliação sensorial. 2.ed. Curitiba: CEPPA, 1984. $101 \mathrm{p}$.

MOOZ, D.T.; CASTELUCCI, A.; SPOTO, M. Potencial Tecnológico e Alimentício de frutos de Macaúba Acromia Aculeata (jacq).
Lodd. Revista Brasileira de Pesquisa em Alimentos, v.3, n.2, p.86-89, 2012.

OLIVEIRA, S.N.; RODRIGUES, M. Papel da análise sensorial como ferramenta de apoio no processo de desenvolvimento de produtos alimentícios. Associação Brasileira de Educação Agrícola Superior - ABEAS, v.26, n.1, p.40-44, 2011.

PREICHARDT, L.D.; VENDRUSCOLO, C.T.; GULARTE, M.A.; MOREIRA, A.D.S. Efeito da goma xantana nas características sensoriais de bolos sem glúten. Revista Brasileira de Tecnologia Agroindustrial, v.3, n.1, p.70-76, 2013.

RAMOS, M.I.L.; RAMOS FILHO, M.M.; HIANE, P.A.; BRAGA NETO, J.A.; SIQUEIRA, E.M.A. Qualidade nutricional da polpa de Bocaiúva Acrocomia aculeata (Jacq.) Lodd. Ciência e Tecnologia de Alimentos, v.28, n.1, p.90-94, 2008.

REIS, R.C.; ARRUDA, R.M.; ZANELLA, M.S.; JESUS, E.M.; BORSATO, AV. Utilidades da bocaiúva (Acrocomia aculeata (Jacq.) Lodd. ex Mart) sob o olhar da comunidade urbana de Corumbá, MS. Cadernos de Agroecologia, v.7, n.2, p.1-4, 2012.

RESURRECCION, A.V.A. Consumer sensory testing for product development. Gaithersburg: Aspen Publishers Inc., 1998. 255p.

ROCHA, M.S.; FIGUEIREDO, R.W.; ARAÚJO, M.A.M.; MOREIRA-ARAÚJO, R.S.R. Caracterização físico-química e atividade antioxidante (in vitro) de frutos do cerrado Piauiense. Revista Brasileira de Fruticultura, v.35, n.4, p.933-941, 2013.

SANJINEZ-ARGANDOÑA, J.E.; CHUBA, M.A.C. Caracterização biométrica, física e química de frutos da palmeira bocaiuva Acrocomia aculeata (Jacq) Lodd. Revista Brasileira de Fruticultura, v.33, n.3, p.1023-1028, 2011. 
TABELA BRASILEIRA DE COMPOSIÇÃO

DE ALIMENTOS (TACO). Tabela

Brasileira de Composição de Alimentos. $2^{\text {a }}$

ed. Campinas: NEPA/UNICAMP, 2006.

$114 p$.

TEIXEIRA, E.; MEINERT, E.M.;

BARBETTA, P.A. Análise sensorial de

alimentos. Florianópolis: Editora da UFSC,

1987. 180p.

VEIGA NETO, A.R.; MELO, L.G. Factors

influencing children's food purchasing

behavior. Saúde e Sociedade, v.22, n.2,

p.441-455, 2013. 